\title{
Modernité, individualisme et crise de l'École
}

\author{
Marie-Hélène Dubost
}

Dans Le Télémaque 2010/1 ( N $^{\circ}$ 37), PAGeS 125 À 136

Éditions Presses universitaires de CAEN

ISSN 1263-588X

ISBN 9782841333585

DOI 10.3917/tele.037.0125 


\title{
ÉTUDE
}

\section{Modernité, individualisme et crise de l'École}

\begin{abstract}
Résumé: Alors que la conception humaniste classique envisageait l'homme comme sujet réflexif trouvant dans son autodétermination le fondement de sa liberté et de sa responsabilité dans le monde, l'individualisme contemporain, en privilégiant les valeurs et les éléments de la sphère privée, enferme l'homme dans les bornes de sa propre indépendance. Dans une société ainsi atomisée, déjà dénoncée par Durkheim, tout projet d'éducation semble impossible, a maxima défini par la coexistence pacifique et tolérante des individus entre eux, une socialisation élémentaire et l'épanouissement personnel. Pour l'homme de l'individualisme, l'éducation se décline alors en termes de potentiel et de formation incessante, "tout au long de la vie", l'éducation civique elle-même visant plus à inculquer des savoir-être qu'à éveiller l'apprentissage de la raison.
\end{abstract}

Le thème de l'individualisme, à l'aune duquel tend à être analysé assez communément désormais le malaise de l'École contemporaine, peut nous apparaître récent. Il semble bien pourtant que la thématique s'ancre plus anciennement, et dès la fin du XIX ${ }^{\mathrm{e}}$ siècle, dans la problématique d'une École confrontée à la mise à l'épreuve de son adaptation aux valeurs de cette société nouvelle qui s'imposent dans le sillage de la III ${ }^{e}$ République et que porte la démocratie libérale. Se pencher sur cette histoire est une des manières d'éclairer le débat actuel sur la relation entre crise de la Modernité, représentations de l'enfant et crise de l'École.

\section{Du sujet à l'individu}

Sans prétendre réécrire ici l'histoire de l'individualisme, il n'est pas inutile de rappeler combien la substitution progressive de l'individu ${ }^{1}$ au sujet renouvelle le champ des idées qui gouvernent tout aussi bien notre rapport au monde que notre rapport à l'apprendre et à l'éduquer.

En effet, comme le montre Alain Renaut ${ }^{2}$, l'archétype individualiste tend à se construire sur le modèle théorique de la monade leibnizienne ${ }^{3}$ qui, dans sa constitution comme dans ses modalités d'existence, dès lors qu'elle n'entretient pas de relation à l'autre, n'est limitée que d'elle-même, sans aucune prise sur l'ordre

1. «Individu», emprunté au latin individuum, «ce qui est indivisible».

2. A. Renaut, «Heidegger. Le règne du sujet» et «Leibniz. L'idée monadologique et la naissance de l'individu », in L'ère de l'individu, Paris, Grasset, 1989, p. 27-68 et 115-151.

3. G.W. Leibniz, La Monadologie, 1714, en particulier $\$ 7$ et $\$ 10$. 
des choses. Parce que le cogito monadologique n'est ni auto-fondé ni auto-réflexif, mais indépendant et auto-référencé, il apparaît comme le fondement ontologique d'un individualisme dont l'autosuffisance fait de l'indépendance son mode d'être collectif, forclusion de toute véritable construction du sujet, reprenant à son compte la dynamique existentielle de la monade comme «auto-déploiement de sa déterminité propre ${ }^{4}$.

Un siècle plus tard, Benjamin Constant, dans un discours prononcé en 1819 à l'Athénée royal de Paris, «De la liberté des Anciens comparée à celle des Modernes", oppose à la liberté des Anciens, liberté collective tournée vers la participation aux affaires de la Cité, la liberté des Modernes, tournée au contraire vers «l'indépendance privée», affichant son refus de tout assujettissement au corps collectif et occultant toute référence primordiale au lien social.

Mais, précise encore Alain Renaut ${ }^{5}$, le glissement de sens qui affecte l'idée de liberté occulte le moment de l'autonomie, préalable pourtant indispensable à cette indépendance qui présuppose la subjectivité comme source de la souveraineté nationale puisqu'au principe de la démocratie, chacun ne doit être soumis qu'à la loi qu'il s'est donnée. En sorte qu'il y a non pas un mais deux épisodes de la liberté chez les Modernes dont le second masque le premier. L'autonomie humaniste, d'abord, s'affirme comme capacité d'auto-fondation lorsque s'énonce, avec Rousseau et Kant, la question de la liberté en termes d'arrachement à toute détermination naturelle. L'indépendance individualiste, ensuite, déclare sa focalisation sur les modalités de l'existence particulière et tend à brouiller la légitimité de toute appréhension normative, finalement perçue comme une limitation de l'affirmation pure du Moi, nouvelle valeur imprescriptible.

À la normativité de l'autonomie, consubstantielle à la construction du sujet, cherche donc à se substituer l'authenticité spontanée du souci de soi coextensive à la présence immédiate de l'individu, dans un contexte où l'opposition public/ privé survalorise les bonheurs privés avec, pour conséquence, la désertion de l'espace public déjà décrite par Alexis de Tocqueville ${ }^{6}$. Lointain écho de la monade leibnizienne, la définition tocquevillienne de l'individualisme soutient en effet l'idée d'une société d'individus atomisés dans laquelle se développe

[le] sentiment réfléchi et paisible qui dispose chaque citoyen à s'isoler de la masse de ses semblables et à se retirer à l'écart avec sa famille et ses amis, de telle sorte que, après s'être ainsi créé une petite société à son usage, il abandonne volontiers la grande société à elle-même ${ }^{7}$.

Ainsi, à l'indépendance et à l'autosuffisance d'individualités dont la nature s'accomplit selon une genèse développementaliste vient s'ajouter, lorsque la réflexion

4. A. Renaut, L'ère de l'individu, p. 138.

5. A. Renaut, «Heidegger. Le règne du sujet».

6. A. de Tocqueville, «De l'individualisme dans les pays démocratiques» [1840], in De la Démocratie en Amérique, t. 2, Paris, Pagnerre, 1848, II, 2.

7. A. de Tocqueville, De la démocratie en Amérique, t. 3, II, 2, p. 196. 
se déporte de l'ontologique au politique, la coexistence et la valorisation des sphères privées.

Par conséquent, la centration sur l'individu comme principe de tout arbitrage brise ici la Modernité. À la conception humaniste par laquelle l'humanité est pensée par référence à un sujet auto-réflexif et auto-fondé, dans une tension vers l'autonomie (la liberté), tend à se surimposer une représentation nouvelle par laquelle l'humanité est rapportée à l'individu autoproclamé, mesure de toute chose, dont les «valeurs» qui lui sont immanentes sont explicitement limitées à la sphère de l'intérêt et de l'inclination individuelle.

Alors que l'homme de l'humanisme n'entend recevoir de loi, ni de la nature des choses, ni de Dieu, mais de sa raison et de sa liberté, et ainsi affirmer le fondement (sub-jectum) de ses représentations et de ses actes, l'homme de l'individualisme ne peut outrepasser les bornes de sa propre indépendance par laquelle il agit nécessairement à partir de lui-même et pour lui-même, selon son intérêt particulier.

Sans doute Hegel anticipe-t-il le danger:

Ce que les récents bouleversements du temps ont si souvent amené: l'indifférence, le désespoir et la perte de la croyance, ailleurs si puissante, que le citoyen peut agir efficacement, aussi à la place qui est la sienne, pour le bien général - un tel spectacle de l'expulsion de l'intérêt pour ce qui touche la communauté et pour la vie publique qui est allée à l'abîme, peut éveiller des sentiments plus douloureux que le spectacle des cadavres laissés par des cités et des ruines de murailles et de demeures autrefois célèbres ${ }^{8} \ldots$

Et Ferdinand Buisson, un des pères de l'école ferryste, rappelle que «nous sommes un peuple et non pas une réunion désordonnée d'individus égoïstes et sans lien ${ }^{9}$. À cette prégnance de l'individualisme fait encore résonance cette réflexion de Jean-Paul Sartre:

Avant la guerre je me considérais simplement comme un individu, je ne voyais pas du tout le lien qu'il y avait entre mon existence individuelle et la société dans laquelle je vivais. Au sortir de l'École normale, j'avais bâti toute une théorie là-dessus: j'étais «l'homme seul», c'est-à-dire l'individu qui s'oppose à la société par l'indépendance de sa pensée mais qui ne doit rien à la société et sur qui celle-ci ne peut rien, parce qu'il est libre ${ }^{10} \ldots$

Mais de la part de ceux-là mêmes qui s'alarment bientôt, les interprétations de la situation, une fois passée l'incantation, revêtent un caractère multiple, polymorphe et contradictoire, qui augure d'une sérieuse inflexion quant à la définition des fins de l'éducation et de la mission de l'École.

8. G.W.F. Hegel, «Discours du 9 septembre 1811», in Textes pédagogiques, Paris, Vrin, 1990, p. 104.

9. F. Buisson, La Foi laïque: extraits de discours et d'écrits, 1878-1911, Paris, Hachette, 1911, p. 16.

10. J.-P. Sartre, Situations X. Politique et autobiographie, Paris, Gallimard, 1976, p. 177. 


\section{Pluralité des individualismes... et difficulté à penser l'École en termes de projet politique}

Face au risque d'atomisation sociale, Émile Durkheim par exemple, hanté par la menace d'anomie ${ }^{11}$, donne l'alarme, et fait de la socialisation scolaire la priorité car «la société ne peut vivre que s'il existe entre ses membres une suffisante homogénéité ${ }^{12}$. Aussi l'éducation, et singulièrement l'éducation scolaire, a-t-elle

pour objet de susciter et de développer chez l'enfant un certain nombre d'états physiques intellectuels et moraux que réclament de lui et la société politique dans son ensemble et le milieu spécial auquel il est particulièrement destiné ${ }^{13}$ [car] la société dépasse l'individu ${ }^{14}$.

Mais, annonce-t-il,

que la société s'oriente dans un sens individualiste, et tous les procédés d'éducation qui peuvent avoir pour effet de faire violence à l'individu, de méconnaître sa spontanéité interne apparaîtront comme intolérables et seront réprouvés ${ }^{15}$.

Pour autant, ce primat de la société est encore posé par Durkheim au nom de la valeur de la personne. C'est parce que

[l'] on s'achemine ainsi peu à peu vers un état, qui est presque atteint dès maintenant, et où les membres d'un même groupe social n'auront plus rien de commun entre eux que leur qualité d'homme, que les attributs constitutifs de la personne humaine en général [qu'] il n'y a pas de raison d'État qui puisse excuser un attentat contre la personne quand les droits de la personne sont au-dessus de l'État ${ }^{16}$.

Cependant, la menace d'une fragmentation sociale portée par un individualisme "dissolvant de l'union nationale ${ }^{17}$ est aussi prétexte à une formidable croisade antimoderne, d'inspiration traditionaliste et antihumaniste. L'École en est le théâtre et l'universalisme kantien la cible privilégiée lorsque, jusqu'à la Seconde Guerre mondiale, les maîtres républicains sont accusés de penser l'homme «en soi» contre l'homme du concret, l'homme de sa famille, de sa terre, de sa patrie ou de sa race.

C'est ici Maurice Barrès qui, dans le premier volume de sa trilogie du Roman de l'énergie nationale, Les Déracinés (1897), fait d’un professeur de philosophie

11. É. Durkheim, Le suicide: étude de sociologie [1897], Paris, PUF, 1967.

12. É. Durkheim, «L'Éducation: sa nature, son rôle», in Éducation et sociologie [1911], Paris, Alcan, 1922 , p. 48

13. Ibid., p. 49.

14. É. Durkheim, L'Éducation morale, cours de sociologie dispensé à la Sorbonne en 1902-1903, Paris, Alcan, 1934, p. 50.

15. É. Durkheim «Pédagogie et sociologie» [1893], in Éducation et sociologie, p. 130.

16. É. Durkheim, La Science sociale et l'Action [1898], Paris, PUF, 1987, p. 255 et 258.

17. Selon la formule de Ferdinand Brunetière, directeur de la Revue des deux mondes au moment de l'affaire Dreyfus, cf. M. Winock, Le siècle des intellectuels, Paris, Seuil, 1997, p. 48. 
kantien, Paul Bouteiller, l'incarnation même de l'ennemi national ${ }^{18}$. Et c'est là Charles Maurras pour, en pleine affaire Dreyfus, commenter auprès de Barrès la fameuse pétition des «intellectuels» :

Avez-vous vu la protestation de ce ramas de juifs, de huguenots, et de néo-kantiens? Car c'est bien le kantisme et l'université criticiste qui servent de ciment à tous les intellectuels ${ }^{19} \ldots$

Car, et même en prenant du champ par rapport à cette droite nationaliste et antirépublicaine, l'universalisme, d'esprit cosmopolitique, est souvent tenu responsable de la dissolution du lien social et de la déchristianisation, de l'avènement

[de l'] individu anonyme, insexuel, sans ancêtres, sans tradition, sans milieu, sans lien d'aucune sorte, voilà, - Taine l'avait prévu, - l'homme de la fausse démocratie, celui qui vote et dont la voix compte pour un, qu'il s'appelle Thiers, Gambetta, Taine, Pasteur, ou qu'il s'appelle Vacher. L'individu finira par rester seul avec son moi, à la place de tous les «esprits collectifs», à la place de tous les «milieux professionnels» qui avaient, à travers le temps, créé des liens de solidarité et maintenu des traditions d'honneur commun. Ce sera le triomphe de l'individualisme atomiste, c'est-à-dire de la force, du nombre et de la ruse.

\section{Aussi convient-il de défendre}

un certain mode d'éducation hiérarchique [pour] faire ainsi triompher la conception organique de la société sur la conception purement contractuelle et atomique, empêcher l'universelle pulvérisation de ce grand corps qui est la patrie ${ }^{20}$.

Si les termes de ce débat sont assurément datés, il n'est pas certain que les idées le soient autant. Elles s'expriment sporadiquement à travers un discours nostalgique

18. La métonymie est grossière. Sept jeunes Lorrains, nés vers 1860, rencontrent en M. Bouteiller, leur professeur de philosophie, l'héritier de l'universalisme des Lumières. Celui-ci les incite à quitter la Lorraine pour Paris, capitale des Sciences, selon le principe que l'éducation élève, et donc arrache à sa condition particulière pour accéder à l'abstraction du concept, à l'universelle raison. Pour les héros, la rupture avec leur terroir et leur famille sera tragique...

19. Souligné par Maurras et cité par M. Winock, Le siècle des intellectuels, p. 89. On pourrait également évoquer, quant à cette question, Ferdinand Brunetière (1849-1906), directeur de la Revue des deux mondes au moment de l'affaire Dreyfus, pour qui l'individualisme est un «dissolvant de l'union nationale». Rationaliste, agnostique et anti-intellectualiste - au sens où il s'oppose à l'intervention des intellectuels dont l'individualisme aristocratique révèle l'impéritie à se hisser au niveau de l'intérêt national -, Brunetière est persuadé de la nécessité d'une Église forte contre la menace de la décomposition sociale, et s'engage à ce titre dans le combat de l'anti-dreyfusisme sans être antisémite (ibid., p. 48).

20. A. Fouillée, La Démocratie politique et sociale en France, livre III, «L'enseignement dans la démocratie», Paris, Alcan, 1923, p. 131-132. Précisons qu'en dépit des apparences peut-être, Alfred Fouillée (1838-1912), essayiste et professeur de philosophie à l'École Normale Supérieure, est tenu par Durkheim, qui fait une sévère critique de son ouvrage, La propriété sociale et la démocratie (1884), pour un «conciliateur», habile à réaliser la synthèse entre des positions adverses, et non pour représentant de la droite radicale (É. Durkheim, "Alfred Fouillée, La propriété sociale et la démocratie», Revue philosophique, XIX, 1885, p. 446-453). 
tentant de ressusciter une école qui savait hier, assure-t-on un peu vite, former les meilleurs et rassembler autour des valeurs du mérite, de l'effort et de l'identité nationale. Et elles se survivent dans la volonté de restaurer les compétences sociales et civiques, particulièrement dans le «Socle commun de connaissances et de compétences», "ciment de la Nation» que le décret du 11 juillet 2006 met en scène ${ }^{21}$, actualisant l'article 2 de la loi d'orientation du 23 avril 2005: "la nation fixe comme mission première à l'école de faire partager aux élèves les valeurs de la République».

Or, pour cette société démocratique où l'individu s'impose en effet à partir de l'effacement du sujet et s'affirme comme indépendance absolue, sorte de primat normatif par quoi «lui seul vaut», le problème de penser des valeurs transcendantes à l'individualité reste entier. Dès lors, il devient difficile de décider d'un projet collectif excédant la cohabitation pacifique, respectueuse de l'existence de chacun reconnu dans son égale dignité, difficile d'appréhender un mode de l'être-ensemble, de définir des normes communes, sans lesquelles pourtant il n'y a plus de république et finalement plus d'indépendance. Au point, d'ailleurs, que les théoriciens de l'idéologie libérale y renoncent par hypothèse, substituant à la transcendance normative l'immanence d'un optimum social harmonieux qui procède immédiatement de la libre confrontation des intérêts égoïstes. Confer la métaphore smithienne de la «main invisible», par le jeu de laquelle nous œuvrons sans le devoir, puisque sans le savoir ni le vouloir, à l'intérêt de tous ${ }^{22}$.

\section{Émergence d'une nouvelle figure de l'École comme fer de lance d'une émancipation sociale}

Dans un tout autre registre politique - et bien qu'individualisme et égoïsme renvoient à des champs sémantiques distincts ${ }^{23}$-, l'individualisme libéral comme triomphe de l'égoïsme des intérêts particuliers se trouve mis en cause comme sont revendiquées l'abolition des privilèges de naissance et l'amélioration du sort des plus pauvres. Ce jugement moral, très présent chez les saint-simoniens et les proto-socialistes dès le $\mathrm{XIX}^{\mathrm{e}}$ siècle, marque tout le $\mathrm{XX}^{\mathrm{e}}$ siècle.

Prenant le contre-pied d'un tel égoïsme social, le volontarisme d'un parti pris antiélitiste engagé dans un combat pour la démocratie de l'enseignement prend alors le relais du mouvement d'émancipation éducative et sociale d'inspiration

21. Décret $\mathrm{n}^{\circ} 2006830$ du 11 juillet 2006 relatif au socle commun de connaissances et de compétences, Bulletin officiel de l'Éducation nationale $\mathrm{n}^{\circ} 29$ du 20 juillet 2006.

22. A. Smith, Recherches sur la nature et les causes de la richesse des nations [1776], Paris, Flammarion, 1991, vol. 1, livre I.

23. Distinction sur laquelle Tocqueville insiste justement: «L'individualisme est une expression récente qu'une idée nouvelle a fait naître. Nos pères ne connaissaient que l'égoïsme. L'égoïsme est un amour passionné et exagéré de soi-même, qui porte l'homme à ne rien rapporter qu'à lui seul et à se préférer à tout. [...]. L'égoïsme naît d'un instinct aveugle; l'individualisme procède d'un jugement erroné plutôt que d'un sentiment dépravé». A. de Tocqueville, "De l'individualisme dans les pays démocratiques», p. 195-196. 
rousseauiste porté par des pédagogues comme Johann Pestalozzi ou Friedrich Fröbel $^{24}$, et du mouvement caritatif chrétien favorable à la scolarisation des pauvres. De ce mouvement est d'ailleurs redevable le développement de l'enseignement préélémentaire, de la salle d'asile à la maternelle ${ }^{25}$, et pour partie tout au moins, de l'enseignement populaire ${ }^{26} \mathrm{du} \mathrm{XIX}^{\mathrm{e}}$ siècle à nos jours.

Du principe de l'égalité à l'égalité démocratique, le mode de légitimité du contemporain continue de s'imposer à l'École tout au long du $\mathrm{XX}^{\mathrm{e}}$ siècle, et l'injonction se fait de plus en plus pressante. Sans doute l'École est-elle sommée de se démocratiser effectivement, mais, en réponse aux exigences où s'emmêlent équité et égalité, elle doit assurer désormais la réussite personnelle de chaque enfant.

Ce constat appelle deux remarques.

D'abord, l'idée d'une fonction régulatrice de l'École dans le champ social est loin d'aller de soi. Non seulement elle est étrangère aux fondements de l'École moderne issue des Lumières - le sujet, la raison critique, l'histoire -, dont la mission ambitionne d'assurer, par le savoir, l'égalité politique des individus et non de leur permettre de compenser leurs "handicaps sociaux», mais elle est incompatible avec cette exclusive politique dès lors qu'il s'agit non pas seulement de construire des sujets-citoyens mais de former des individus privés. L'enjeu, d'un point de vue moderne, n'est pas en effet la réussite de chacun par l'École, ni même la démocratisation de la sélection des élites, mais la réussite de l'institution démocratique qu'est l'École ${ }^{27}$. Celle-ci doit être évaluée sur son aptitude à instituer l'homme comme citoyen libre, car, et Ferdinand Buisson le dit très simplement, «l'école [...] ne fait pas les élections, mais elle fait les électeurs ${ }^{28}$, thématique dont il faut bien convenir qu'elle a pratiquement disparu de notre paysage idéologique ${ }^{29}$. Libre, c'est-à-dire libéré de l’ignorance, primat réaffirmé par Alain:

Il reste ceux que l'on n'instruit guère, soit parce qu'ils ne veulent pas apprendre, soit parce qu'ils ne peuvent. Ici se trouve le problème véritable. J'ai connu un temps où le jeune garçon qui raisonnait mal une fois ou deux sur les triangles était aussitôt abandonné. Conduite raisonnable, si le pouvoir ne cherche que des recrues pour la partie

24. Ces pédagogues ajoutent à l'émancipation humaniste par l'école l'idée d'une émancipation sociale: c'est désormais par l'école que peut être amélioré le sort des plus pauvres.

25. J.-N. Luc, L'invention du jeune enfant au XIXe siècle, Paris, Belin, 1997.

26. Ce faisant, et même si des intentions moins charitables s'immiscent dans le cahier des charges de ces écoles, en termes de maintien de l'ordre et de formation de main-d'œuvre, cette orientation caritative, traversée d'ambiguïté sans doute, s'inscrit dans le mouvement historique de la scolarisation et sur la part prise par l'Église dans cette histoire.

27. C'est explicitement la position de Condorcet par exemple, et, implicitement, de Rousseau. Cf. lectures croisées du Contrat social (1762), de l'Émile (1762) et des Considérations sur le gouvernement de Pologne et sur sa réformation projetée (1770-1771), en particulier du chapitre IV, "Éducation", in Euvres complètes, Paris, Gallimard (Pléiade), t. III (1964), p. 966.

28. F. Buisson, «Un discours en Vendée» (1887), in La Foi laïque..., p. 40.

29. Selon l'enquête commandée par le SNUIPP à l'institut de sondage CSA pour la rentrée 2007, seulement $30 \%$ des personnes interrogées estiment que la formation du futur citoyen est une priorité de l'école, cf. Fenêtres sur cour n 301 du 10 septembre 2007, p. 12. 
gouvernante; conduite ridicule, si le pouvoir veut réellement des citoyens éclairés. [...] Il n'y a point d'homme, évidemment, dont je puisse annoncer qu'il ne pensera pas au-delà de son métier. Quand il serait esclave comme Esope, il pensera encore. Or il ne sera pas esclave. Non seulement il pensera aux choses divines et humaines, tant bien que mal, comme chacun fait, mais, bien plus, il décidera de la paix et de la guerre, du juste et de l'injuste, de noblesse, de bassesse, et enfin de tout $[\ldots]^{30}$.

En quoi un des aspects de la crise de la modernité scolaire, dont hérite l'École de ce début de XXI ${ }^{\mathrm{e}}$ siècle, passe bien par un recadrage des tâches assignées à celleci, recadrage par lequel socialisation élémentaire et épanouissement de l'individuenfant tendent à se substituer à l'émancipation par l'instruction de l'élève, du sujet citoyen.

Ensuite, une telle (re)configuration individualiste des fins de l'éducation scolaire n'est pas sans effets contradictoires qui participent, avec une efficace croissante, au brouillage des missions de l'École.

Cette reconfiguration contribue notamment à ce que l'École, de plus en plus attentive aux sollicitations contemporaines dont elle fait l'objet tant en termes de qualification que de loisirs culturels, laisse finalement dans l'ombre un rapport au savoir qui ne porte plus aucune promesse émancipatrice au plan politique. Or, comme l'ont amplement montré les travaux du laboratoire ESCOL, ce rapport qui, pour les enfants des milieux populaires, n'est construit qu'à l'école, demeure indispensable à la réussite scolaire de chacun ${ }^{31}$. En sorte que cette société, qui n’a effectivement jamais été aussi scolarisée ni aussi instruite, échoue devant la démocratisation de l'accès aux savoirs et de la sélection des élites, et secrète idéologiquement les conditions d'une certaine régression intellectuelle, sans pouvoir pour autant se porter garante de l'épanouissement de l'individu-enfant.

En outre, une appréhension de la ségrégation, de l'exclusion, de la marginalisation scolaires solidaire, aujourd'hui encore, d'une représentation anciennement compassionnelle et caritative de l'École, joue dans le même sens. L'idéologie des Droits de l'Homme ${ }^{32}$ qui, en toute logique... individualiste, substitue la réparation des injustices subies par les individus à la recherche d'une justice entre groupes sociaux, et subroge une justification immanente en la justification transcendante de l'organisation collective, fait place nette au bon cœur et à l'enthousiasme protestataire face au sensationnalisme de l'inacceptable ${ }^{33}$.

30. Alain, «Propos XX», in Propos sur l'éducation [1932], Paris, PUF, 1948, p. 38.

31. B. Charlot, É. Bautier, J.-Y. Rochex, École et savoir dans les banlieues et ailleurs, Paris, Armand Colin, 1992.

32. Si les Droits de l'homme conservent évidemment un point de vue normatif, ils restent muets quant au pourquoi de ce qui est, et discréditent même toute volonté de le comprendre dès lors qu'ils lui opposent la prise de conscience morale d'intervenir dans l'urgence. Ainsi est recyclée l'ancienne critique sociale dont on n'attend plus qu'elle formule une explication, encore moins une solution globale.

33. M. Gauchet, "Quand les droits de l'homme deviennent une politique », Le Débat, $\mathrm{n}^{\circ}$ 110, mai-août 2000. 
Ainsi, pour les enseignants en $\mathrm{ZEP}^{34}$, «les enfants de pauvres» sont souvent regardés comme «de pauvres enfants ${ }^{35}$. Et parmi les (mauvaises) raisons pour baisser le niveau d'exigence scolaire, et donc renoncer à l' "ambition de savoir et de culture» autant qu'à l'aspiration à l'égalité, figure en bonne place ${ }^{36}$ le souci de faciliter la tâche à ces élèves en grande détresse sociale en aplanissant les difficultés afin de leur éviter une nouvelle épreuve. Par quoi l'enfer est pavé de bonnes intentions car ce faisant, toute démocratisation de l'accès au savoir et à l'exercice critique du savoir est impossible ${ }^{37}$.

\section{Éduquer l'individu?}

Mais parallèlement à ces équivoques que conjecture directement ou indirectement la projection individualiste, l'omnipotence d'un individu pour l'essentiel désinvesti de la charge sociale que l'éducation, par hypothèse, incorpore à la construction du sujet, laisse l'École désemparée.

Car d'une part, dans sa logique ultime, l'homme de l'individualisme qui s'accomplit n'a plus rien à devenir en soi : il est déjà... ce qu'il est.

Précisément, et contrairement à ce que l'on entend parfois, il n'est pas autofondé puisqu'il n'y a ni volonté libre ni morale de l'intention qui préside à ses actes, et qu'aucune instance ne gouverne, en raison, l'élaboration de son rapport à l'Autre. L'individu est saisi dans la toute-puissance de son autosuffisance, et l'assertion de son indépendance. Comme le dit Marcel Gauchet,

pour un individu qui est posé et qui se pose toujours déjà là, préalablement à toute acquisition, il n'y a en vérité qu'une auto-éducation possible; il n'y a qu'une autoproduction qui soit imaginable ${ }^{38}$.

Et que cet individu réclame toujours plus de formation, afin de se réaliser en s'identifiant culturellement et, plus sûrement encore, en accédant à une place sociale

34. Les Zones d'éducation prioritaire sont mises en place par les circulaires du $1^{\text {er }}$ juillet et du 28 décembre 1981 sur le principe d'une pédagogie de la compensation, idée américaine des années 1920-1930, discrimination positive déjà expérimentée en Grande-Bretagne dans les années 1960. Face à l'échec des solutions centrales et standardisées, l'État tente la solution locale dans un contexte de décentralisation (1982-1983), de valorisation des pédagogies de projet et d'ouverture de l'école sur son environnement, en convergence avec le développement social des quartiers.

35. J.-Y. Rochex, "Ambition de savoir et de culture, exigence d'égalité et enjeux de solidarité » et "La "théorie" du handicap socio-culturel: une explication ethnocentriste qui n'explique rien", Dialogue, n 96-97, printemps-été 2000, Pratiques de savoir en banlieue.

36. L'entretien de la motivation par l'attractivité des activités proposées, et la réduction de la distance culturelle entre enseignants et enseignés par le maintien du lien avec la culture familière des élèves doivent également être évoqués, et participent du reste de la même représentation de la scolarisation des enfants de milieux populaires.

37. J.-Y. Rochex, «À l'école: réhabiliter le travail intellectuel», Dialogue, n 94, automne 1999, Mais où sont passés les savoirs?.

38. M. Gauchet, «Démocratie, éducation, philosophie», in M.-C. Blais, M. Gauchet, D. Ottavi, Pour une philosophie politique de l'éducation, Paris, Hachette littératures (Pluriel Philosophie), 2003, p. 42. 
optimale, est peut-être moins le signe d'une forte demande d'éducation, hypothèse que Marcel Gauchet envisage ${ }^{39}$, que d'une éducation impossible. "Impossible», du moins, au regard de ce qu'est l'éducation dans son acception moderne, historiquement partie prenante de la constitution de l'École à partir de la Renaissance, et pour laquelle "L'homme ne peut devenir homme que par l'éducation " ${ }^{40}$.

D'autre part, l'École contemporaine est tentée d'intégrer à son fonctionnement la représentation d'une société cloisonnée, partagée entre la sphère publique (social / collectif) et la sphère privée (particulier / individuel), et d'opposer ainsi logique socialisatrice et logique puérocentriste, éludant l'intergénérationalité qui les unit. Alors que l'enfant qui apprend est renvoyé à son seul fonctionnement cognitif, indépendamment des apprentissages collectifs et conformément aux logiques de l'espace privé, est invoquée, conformément cette fois aux exigences de l'organisation publique, une socialisation politique séparée, isolée, supposée restaurer dans l'urgence cet «esprit civique» qui menace de se perdre, mode de socialisation qui est en réalité ce par quoi l'École renonce implicitement à instituer le sujet-citoyen.

De ce point de vue, l'éducation civique ${ }^{41}$ comme dispositif spécifique et pédagogie mutante qui annonce le règne du débat d'opinion(s) et de la transversalité post-disciplinaire, se révèle à bien des égards exemplaire de cette évolution.

En effet, elle assume de n'être pas "liée à un enseignement mais à tous " ${ }^{42}$. Elle anticipe ainsi, avant la loi d'orientation et de programme pour l'avenir de l'école du 23 avril 2005, la coexistence de deux paradigmes organisateurs du cursus scolaire, disciplinaire et «interdisciplinaire». Cette dualité est bien sûr reprise et renforcée après 2005 avec le maintien d'une programmation/progression disciplinaire et l'imposition, en surplomb, d'un socle commun axé sur les compétences, promues comme fins ultimes d'une scolarité dont les connaissances sont désormais les moyens ${ }^{43}$.

En outre, l'éducation civique renonce, de plus en plus explicitement, à instrumenter la raison - mission par excellence de l'éducation des Lumières - pour

39. «Il veut l'éducation, mais il ne veut pas être éduqué. Autodidacte inculte, il est condamné à une variante du supplice de tantale: il aspire à des savoirs dont il repousse les instruments ", ibid., p. $42-43$.

40. E. Kant, Réflexions sur l'éducation [1803], Paris, Vrin, 1984, p. 73.

41. Résistant vaille que vaille aux diverses crises morales et politiques qui caractérisent la première moitié du XX $\mathrm{XX}^{\mathrm{e}}$ siècle, l'éducation civique, héritière de l'"instruction morale et civique » de l'école ferryste, semble bien emportée, dans les années 1960, par la vague anticonformiste. Le tournant de la décennie voit en effet sa disparition des programmes scolaires du second degré et, dans le premier degré, sa dilution dans les disciplines d'éveil (arrêté du 7 août 1969). Sa restauration par le ministre de l'Éducation nationale Jean-Pierre Chevènement en 1985 dans les écoles et les collèges avec programme, manuel et budget n'en est que plus spectaculaire, d'autant que sa présence est, au cours des vingt années suivantes, confirmée et renforcée puisqu'elle s'impose, sous les traits de l'ECJS (éducation civique, juridique et sociale), entre 2000 et 2002, dans les lycées généraux et professionnels.

42. MEN, Qu'apprend-on à l'école élémentaire?, Paris, CNDP/XO, 2002, p. 177.

43. Décret $n^{\circ} 2006830$ du 11 juillet 2006 relatif au socle commun de connaissances et de compétences. 
formater les consciences lorsqu'elle revendique n'être pas «en priorité, l'acquisition d'un savoir, mais l'apprentissage pratique d'un comportement ${ }^{44}$ et s'assume, dans les programmes de 2008 en cycle 2 et 3 , comme instruction civique et morale ${ }^{45}$. Elle ordonne des priorités parmi lesquelles se disputent à la première place l'épanouissement de l'enfant et la formation de l'acteur social, lors même qu'elle se donne comme la forme la plus évidente, sans certitude quant à son efficience ${ }^{46}$ d'une éducation démocratique à la démocratie.

\section{Conclusion}

Que l'histoire moderne ait fabriqué, à travers la manière dont elle se représente à elle-même, une société d' "individus", selon le modèle proposé par Alain Renaut, interroge donc directement l' "éducabilité" de l'individu-enfant. Confrontées au déploiement de la logique individualiste qui vient briser l'unité de la Modernité, l'École et l'éducation voient peu à peu se lézarder, au cours du $\mathrm{XX}^{\mathrm{e}}$ siècle, les fondations de leur légitimité. Bousculée par la remise en cause de l'héritage moderne qui avait installé l'éducation au cœur du politique, au principe de la genèse du sujet-citoyen, l'École contemporaine multiplie projets, missions et priorités, parfois contradictoires, et balance entre «antepathie» et inflation du nouveau dans les discours éducatifs.

Car dans un monde effectivement désenchanté où, pour parler comme La Rochefoucauld, "l'homme est conduit lorsqu'il croit se conduire " ${ }^{47}$, où tout pourrait demain, ou tout peut déjà peut-être, s'originer, se rapporter, se limiter et se destiner à l'immanence de l'ego et à son principe d'utilité, peut-on encore penser l'éducation et par conséquent l’instruction dans les catégories du Moderne?

Marie-Hélène Dubost

Université de Vincennes - Saint-Denis, Paris VIII

CIRCEFT-ESCOL

44. Ibid.

45. Arrêté relatif aux horaires et programmes d'enseignement de l'école primaire, $B O$ hors-série $n^{\circ} 3$ du 19 juin 2008.

46. Cf. M.-C. Blais, «Civilité, socialisation, citoyenneté. Que peut l'éducation civique?», in Pour une philosophie politique de l'éducation, p. 251-296.

47. F. de La Rochefoucauld, Sentences et maximes de morale, version du manuscrit Liancourt, 1664, maxime 19, in Euvres complètes, Paris, Gallimard (Pléiade), 1964, p. 341. 
